\title{
Cosmic ray origin: Supernova remnants through the electromagnetic spectrum
}

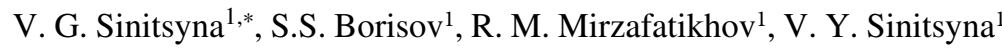 \\ ${ }^{1}$ P. N. Lebedev Physical Institute, Russian Academy of Science
}

\begin{abstract}
Supernova Remnants have long been considered as unique candidates for cosmic-ray sources. Recent observations of several SNRs in X-rays and TeV gamma-rays will help in solving the problem of the origin of cosmic rays and are key to understanding the mechanism of particle acceleration at a propagating shock wave. The observation results of Galactic shell-type supernova remnants at different evolution stages Cas A, Tycho's SNR, $\gamma$ Cygni SNR, IC 443 and G166.0+4.3 by the SHALON mirror Cherenkov telescope are presented. For each SNR the SHALON observation results are given with its spectral energy distribution compared with other experimental data and images by SHALON together with data from X-ray by Chandra and radio-data by Canadian Galactic Plane Survey DRAO (CGPS). The comparison of the source's morphology in different energy bands could reveal its essential features as a forward and reverse shock or the location of swept out dense molecular cloud. The experimental data presented here have confirmed the prediction of the theory about the hadronic generation mechanism of very high energy $800 \mathrm{GeV}-100 \mathrm{TeV}$ gamma-rays in Tycho's $\mathrm{SNR}$, Cas A and IC 443. Also the collected experimental data help to make clear the origin of TeV gamma-ray emission in the SNRs like $\gamma$ Cygni SNR and G166.0+4.3.
\end{abstract}

\section{Introduction}

The investigation of VHE gamma-ray sources by any method, including mirror Cherenkov telescopes, touches on the problem of the cosmic ray origin and, accordingly, the role of the Galaxy in their generation. The SHALON observations have yielded results on Galactic supernova remnants (SNR) of different ages. Among them are: the shell-type SNRs Tycho's SNR, Cas A, IC 443, $\gamma$ Cygni SNR, G166.0+4.3. For each SNR the SHALON observation results are given with its spectral energy distribution compared with other experiments' data and images by SHALON together with data from X-rays by Chandra and radio-data by CGPS. Also the theoretical predictions are shown. The collected experimental data can help to solve the cosmic ray origin.

\section{Cas A supernova remnant (1680)}

Cas A is athe youngest of historical supernova remnant in our Galaxy. The supernova explosion that gave rise to Cas A occurred around 1680. Cas A was observed with SHALON during the 74 hours in the period 2010 - 2014 [1, 2]. The $\gamma$-ray source associated with Cas A was detected above $800 \mathrm{GeV}$ at a level of $18.1 \sigma$ (for the $\gamma$-ray flux and spectrum details see table 1). Fig. 1, top, shows spectral energy distributions of the $\gamma$-ray emission from Cas A by SHALON ( $\Delta$ ) in comparison with theoretical predictions $[3,4]$ and other experimental data (see refs in $[1,2]$ ).

The SHALON image of Cas A in the $0.8-30 \mathrm{TeV}$ is obtained (see Fig. 1, bottom, grey scale). It is compared

\footnotetext{
*e-mail: sinits@sci.lebedev.ru
}

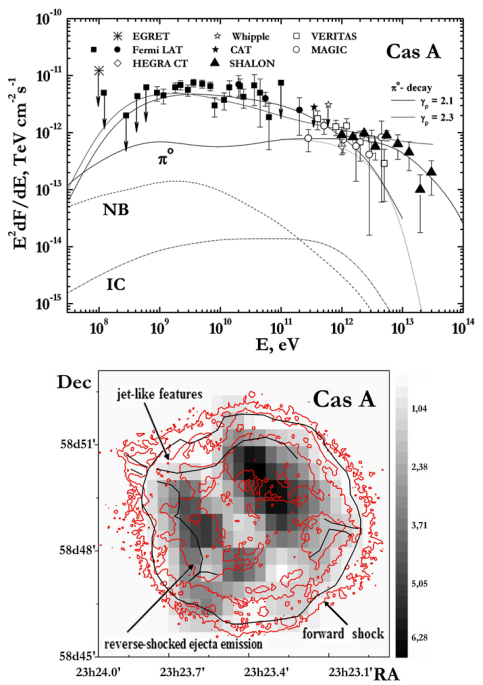

Figure 1. Characteristics of Cas A: top: Spectral energy distributions of high- and very high energy $\gamma$-ray emission by SHALON ( $\boldsymbol{\Lambda})$ in comparison with other experiments. NB - nonthermal Bremsstrahlung gamma-ray energy flux; IC - Inverse Compton $\gamma$-ray energy flux [4]. The red and blue lines are calculations from [3]. bottom: Image of SNR at energies $>0.8 \mathrm{TeV}$ by SHALON (grey scale). Red contours are the X-ray emission by Chandra and show 7\%, $19 \%$ and $54 \%$ of the image maximum.

with the 1.5 - $3.0 \mathrm{keV}$ emission map (Fig. 1, red contours) obtained in observations with the Chandra ACIS [5]. Xray contours are in logarithmic scale. In the analysis of Chandra observations of Cas A [5] it was shown that this SNR has a structure with two remarkable features. One of them is a mostly circular emission at $4-6 \mathrm{keV}$ exiting 

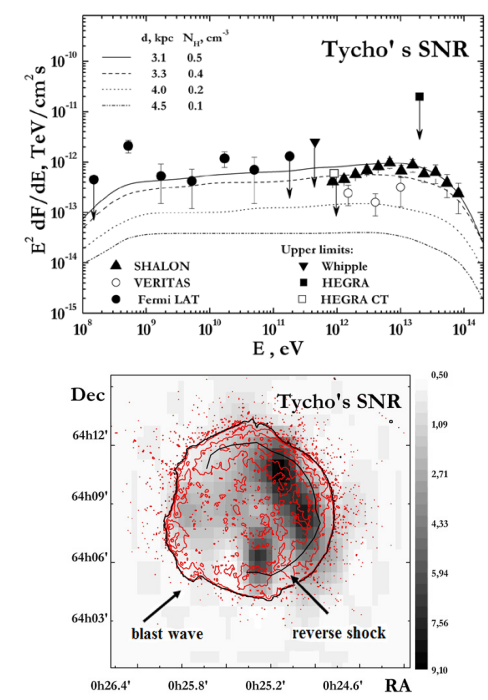

Figure 2. Characteristics of Tycho's SNR:top: Spectral energy distributions of high- and very high energy $\gamma$-ray emission by SHALON ( $\mathbf{\Lambda})$ in comparison with other experiments. Lines show the gamma-ray spectra calculated for four distances $d$ and the corresponding densities of the interstellar medium $N_{H}$. bottom: Image of SNR in the energy range $0.8-80 \mathrm{TeV}$ by SHALON (grey scale). Red contours are the X-ray emission by Chandra. Contour levels are $0.8 \% 2.2 \%, 6.2 \%, 18 \%$ and $51 \%$ of the X-ray emission map maximum.

throughout the remnant and is probably synchrotron emission generated at the forward shock. The location of the forward shock is marked with the outer black contour in Fig. 1. Another one is the reverse-shocked ejecta emission (viewed in 1.78-2.0 keV) with jet-like features in the northeast and in the southwest (viewed in 6.52-6.95 keV). The inner black contour notes the location of this noncircular component. Figure 1, bottom, shows the Chandra $\mathrm{X}$-ray image of Cas A (red lines) with its regions of reverse shock and outer blast wave [5] compared with the TeV structure in the energy range $0.8-30 \mathrm{TeV}$ by SHALON.

The $\gamma$-ray emission in Cas A could be produced via Inverse Compton (IC) scattering and by accelerated cosmic ray hadrons through interaction with the interstellar gas and then $\pi^{\circ}$ - decay. Solid lines in Fig. 1 show the very high energy $\gamma$-ray spectra of hadronic origin [3,4]. It was shown in [3] that the leptonic model with $\mathrm{B}=0.3 \mathrm{mG}$ predicts a 5 - 8 times lower $\gamma$-ray flux than observed; the model with $\mathrm{B}=0.12 \mathrm{mG}$, which can broadly explain the observed $\mathrm{GeV}$ flux, predicts the $\mathrm{TeV}$ spectrum with cut-off energy about $10 \mathrm{TeV}$. The detection of $\gamma$-ray emission at 5 - $30 \mathrm{TeV}$ by SHALON and the hard spectrum below $1 \mathrm{TeV}$ would favor the $\pi^{\circ}$-decay origin of the $\gamma$-rays in Cas A.

\section{Tycho's Supernova Remnant (1572)}

Tycho's SNR originated from the Ia type supernova which exploded in 1572. The supersonic expansion of the stellar debris visible in Tycho's SNR by Chandra has created two $\mathrm{X}$-ray emitting shock waves: one moving outward into the interstellar gas, and another moving back into the debris. Such a character of the displacement of the shock and the contact discontinuity surfaces makes the cosmic ray acceleration at the supernova shock very efficient.
A new source was detected at $\mathrm{TeV}$ energies by SHALON [6] in 1996 with $17 \sigma$ determined by [7]. This object was identified with Tycho's SNRs.

The image of Tycho's SNR obtained in observations by Chandra ACIS in the $0.95-1.261 \mathrm{keV}$ energy bands is presented in Fig 2 with red lines. Red levels are in logarithmic scale to bring out the structure in the remnant. The inner black contour in Fig 2 notes the location of the reverse shock and the outer black contour the location of the blast wave which were determined in paper [8]. The grey scale image in Fig. 2, bottom, shows the TeV structure of Tycho's SNR in the energy range $0.8-80 \mathrm{TeV}$ obtained by SHALON together with the Chandra X-ray emission map. It shows a localization of $\mathrm{TeV}$ emission regions relative to the position of the forward and reverse shock in Tycho's SNR visible in X-rays [8].

Tycho's SNR was also confirmed with VERITAS in observations in the period 2008 - 2010. The $\gamma$-ray emission from Tycho's SNR was detected with Fermi LAT in the range $400 \mathrm{MeV}-100 \mathrm{GeV}$. The expected flux of $\gamma$ quanta from $\pi^{\circ}$-decay, extends up to $>30 \mathrm{TeV}$, while the flux of $\gamma$-rays originated from the IC scattering has a sharp cut-off above a few $\mathrm{TeV}$, so the detection of $\gamma$-rays with energies up to $80 \mathrm{TeV}$ by SHALON [1] (Fig. 2) is an evidence of their hadronic origin. Also, the information on parameters of the Tycho's SNR such as the distance (3.1 - $3.3 \mathrm{kpc}$ ) and interstellar medium density was obtained from the SHALON data within the nonlinear kinetic model [9]. The same parameters have been obtained in calculations of structures visible by Chandra at X-ray energies [10] (see red contours in Fig. 2, bottom).

\section{$4 \gamma$ Cygni SNR (age $\sim(5 \div 7) \times 10^{3}$ years)}

$\gamma$ Cygni SNR is a shell-type supernova remnant, its shelllike features are known in radio- and X-ray energy regions [11]. $\gamma$ Cygni SNR is older then Cas A and Tycho's SNR,

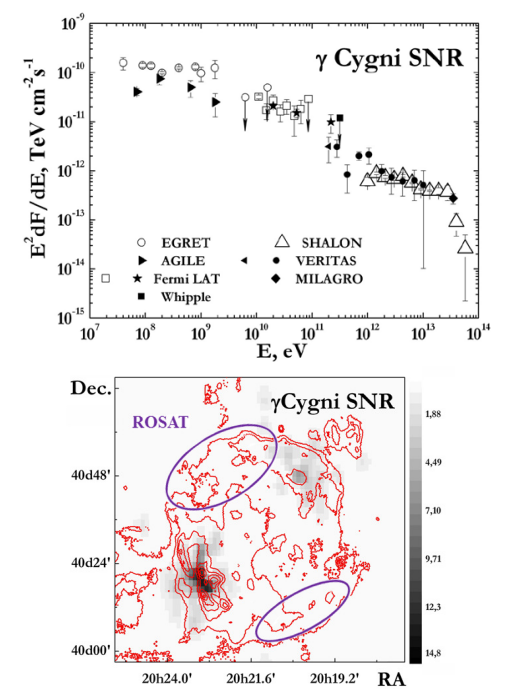

Figure 3. Characteristics of $\gamma$ CygniSNR: top: Spectral energy distributions of high- and very high energy $\gamma$-ray emission by SHALON $(\triangle)$ in comparison with other experiments. bottom: Image of SNR at energies $>0.8 \mathrm{TeV}$ by SHALON (grey scale). Contours are $1420 \mathrm{MHz}$ radio structure by CGPS. Five contour levels are linear scaled and correspond to $10 \%, 20 \%, 30 \%, 40 \%$, $50 \%$ of the map maximum. 


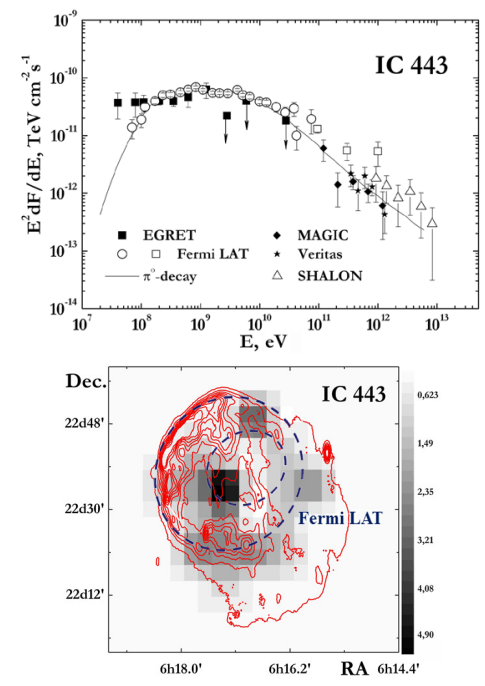

Figure 4. Characteristics of IC443: top: Spectral energy distributions of high- and very high energy $\gamma$-ray emission by SHALON $(\triangle)$ compared with other experiments. The line is the $\gamma$-ray energy flux from $\pi^{\circ}$-decay. bottom: Image of SNR at energies $>0.8 \mathrm{TeV}$ by SHALON (grey scale). Contours are 1420 $\mathrm{MHz}$ radio structure by Canadian Galactic Plane Survey DRAO. Radio contours are linear and correspond to $6.5 \%, 13 \%, 19.5 \%$ and $60 \%$ of the emission map maximum.

and its age is estimated as 5000 - 7000 years [11] and it is supposed to be and in an early phase of adiabatic expansion.

During observations of Cyg X-3 (since 1995) the SHALON field of view contains $\gamma$ Cygni SNR as it is located in the Cygnus Region at $\sim 2^{\circ} \mathrm{SW}$ from Cyg X-3. So due to the large telescopic field of view $\left(>8^{\circ}\right)$ the observations of Cyg X-3 are naturally followed by observations of this SNR. As a result, $\gamma$ Cygni SNR was detected above $800 \mathrm{GeV}$ by SHALON (see $[2,12]$ ). TeV $\gamma$-ray emission regions correlate with the NW and SE parts of the shell visible in radio energies by CGPS (red contours in Fig.3).

An image of $\gamma$ Cygni SNR in the band $0.8-50 \mathrm{TeV}$, obtained using the SHALON long-term observations, is shown in Fig. 3, which also includes the $1420 \mathrm{MHz}$ radio contours of the Canadian Galactic Plane Survey DRAO [13]. The analysis of $\mathrm{TeV} \gamma$-ray arrival directions reveal two emission regions in $\gamma$ Cygni SNR: the main one is at the South-East part of SNR shell and the second one at the North. The main contribution of energy flux is from the South-East region of the SNR shell. These TeV $\gamma$-ray emission regions correlate with the North-West and SouthEast parts of the shell visible at radio energies by CGPS (red contours in Fig.3). The VERJ2019+407 source detected at $200 \mathrm{GeV}$ by VERITAS [14] correlated with the position of the northern part of $\gamma$ Cygni SNR shell.

The visible SNR features observed at X-ray, radio as well as TeV gamma-ray emission can be the result of the presence of shocks at the interaction of the supernova ejecta and the surrounding medium. Very high energy gamma-ray emission in shocks could be produced first via Inverse-Compton scattering of accelerated electrons. Such high energy electrons should also produce Xray synchrotron radiation visible in a non-thermal powerlaw in the X-ray spectrum. But it was shown in [15] that no evidence for a non-thermal component of X-ray spectrum in the North-West part of TeV shell and no source of the X-ray emission were detected at the location of the SE $\mathrm{TeV}$ emission region (see ROSAT for X-rays in Fig. 3).

Also, the $\mathrm{TeV}$ gamma-ray emission can originate from the shock acceleration of hadrons interacting with target material. The density of target material of SNR surroundings estimated in [16] is enough to produce the observable $\mathrm{TeV}$ flux in both regions shown in Fig. 3 via the shock acceleration of hadrons. Thus, the detection of $\gamma$-ray emission at $0.8-50 \mathrm{TeV}$ by SHALON from the North-West and South-East regions of $\gamma$ Cygni SNR would favor the $\pi^{\circ}$-decay origin of the $\gamma$-rays in this SNR.

\section{IC 443 SNR (age $\sim(3 \div 30) \times 10^{3}$ years)}

IC 443 is a shell-type SNR with a complex shape consisting of two half-shells with different radii. IC 443 is one of the best candidates for the investigation of the connection among SNRs, molecular clouds and high- and very high energy $\gamma$-ray sources. The close placement of the dense shocked molecular clouds and detected GeV-TeV $\gamma$ ray emission [17] suggests that IC 443 can be considered as a candidate for the hadronic cosmic-ray source.

IC 443 was detected by SHALON with an integral flux above $0.8 \mathrm{TeV}$ (see table 1, [2, 18] and Fig. 4) with a statistical significance of $9.7 \sigma$ [7]. It was collected during 26 hours of observation. The spectral energy distribution of the $\gamma$-ray emission from IC443 by SHALON $(\triangle)$ compared with other experiments' data EGRET, Fermi LAT, MAGIC, VERITAS [17] and with theoretical predictions is shown in Fig. 4.

The favored scenario in which the $\gamma$-rays of $100 \mathrm{MeV}$ - $10 \mathrm{TeV}$ energies are emitted in the shell of the IC443

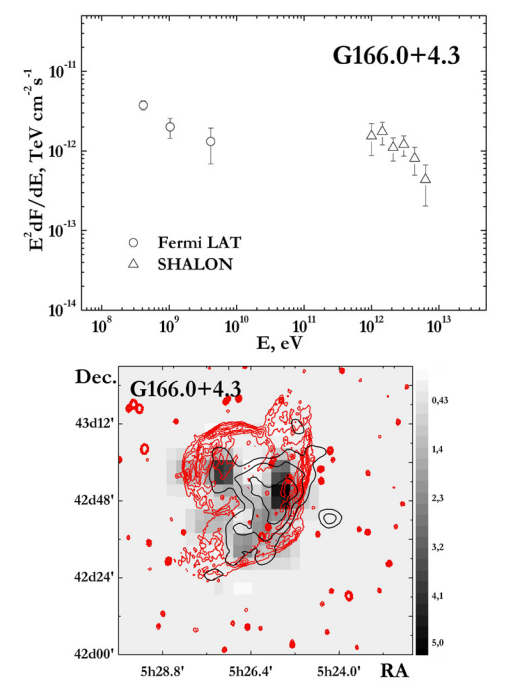

Figure 5. Characteristics of G166.0+4.3: top: Spectral energy distribution of high- and very high energy $\gamma$-ray emission by SHALON $(\triangle)$ in comparison with other experiments. bottom: Image of this SNR at energies $>0.8 \mathrm{TeV}$ by SHALON (grey scale). Red contours are $1420 \mathrm{MHz}$ radio structure by Canadian Galactic Plane Survey DRAO. Contours levels are 10\%, 20\% etc $90 \%$ of the radio emission map maximum. Black contours are Xray structure by ROSAT in the energy range $0.52-2.02 \mathrm{keV}$. Xray contours are logarithmic and correspond to $11 \%, 19 \%, 33 \%$ and $57 \%$ of image maximum. 
Table 1. The catalogue of galactic $\gamma$-ray sources by SHALON with parameters for spectrum fitting in the form of a power law with exponential cutoff $F(>E) \propto E^{k_{\gamma}} \times \exp \left(-E / E_{\text {cutoff }}\right)$.

\begin{tabular}{cccccc}
\hline Sources & Observable flux $^{a}$ & $k_{\gamma}$ & $E_{\text {cutoff }}, \mathrm{TeV}$ & Distance, kpc & Type \\
\hline Cas A & $(0.64 \pm 0.10)$ & $-0.91 \pm 0.11$ & $10.3 \pm 2.5$ & 3.1 & Shell-type SNR \\
Tycho's SNR & $(0.52 \pm 0.04)$ & $-0.93 \pm 0.09$ & $35.0 \pm 5.0$ & $3.1-3.3$ & Shell-type SNR \\
$\gamma$ Cygni SNR & $(1.27 \pm 0.11)$ & $-0.93 \pm 0.09$ & $20.1 \pm 4.2$ & 1.5 & Shell-type SNR \\
IC 443 & $(1.63 \pm 0.23)$ & $-1.94 \pm 0.16$ & - & 1.5 & Shell-type SNR \\
G166.0+4.3 & $(1.49 \pm 0.25)$ & $-1.95 \pm 0.44$ & - & 4.5 & Shell-type SNR \\
\hline \multicolumn{7}{c}{ Integral flux at energy $>800 \mathrm{GeV}$ in units of $10^{-12} \mathrm{~cm}^{-2} \mathrm{~s}^{-1}$} &
\end{tabular}

$\mathrm{SNR}$ is $\pi^{\circ}$-decay produced in the interactions of the cosmic rays with the interstellar gas [17]. The solid line in Fig 4 shows the $\gamma$-ray spectra of hadronic origin. Inverse Compton scattering can not explain the observed IC 443 $\gamma$-ray emission as there is no bright source of seed photons in the region of the IC 443.

In Fig.4, bottom the TeV image of IC443 SNR by SHALON (grey scale) is overlaid with $1420 \mathrm{MHz}$ CGPS DRAO radio contours [13]. The analysis of arrival directions of $\gamma$-rays with energies $800 \mathrm{GeV}-10 \mathrm{TeV}$ reveals the correlation of $\mathrm{TeV} \gamma$-ray emission maxima with $\mathrm{MeV}$ GeV emission observed by Fermi LAT [17] (see Fig. 4), and also $\mathrm{TeV} \gamma$-ray emission of South and South-West parts of IC 443 shell correlated with the position of swept out dense molecular cloud (see red contours by CGPS in Fig. 4).

\section{G166.0+4.3 (age $\sim 24 \times 10^{3}$ years)}

G166.0+4.3 (VRO 42.05.01), a SNR with unusual morphology, contains a shell of circular component in the northeast intersected by a larger bowl-shaped component in the southwest (the wing), expanding into a low density medium [19]. There are important differences in spatial distribution of emission viewed in X-ray and radio energies. The radio image has an outstanding edge-brightened shape in the shell and wing component, whereas the $\mathrm{X}$ ray emission is not edge-brightened, but is dominated by a bright spot in the wing component [19]. As such remnant morphology can be the results from the shock encountering a density discontinuity in the interstellar medium, G166.0+4.3 became a candidate for investigating particle acceleration in SNR shocks at high- and very high energies.

G166.0+4.3 was observed with the SHALON telescope during 20.1 hours in the period 2015 - 2016. The gamma-ray source associated with the G166.0+4.3 SNR was detected above $800 \mathrm{GeV}$ with a statistical significance of $6.1 \sigma$ with a gamma-quantum flux above $0.8 \mathrm{TeV}$ of $(1.49 \pm 0.25) \times 10^{-12} \mathrm{~cm}^{-2} \mathrm{~s}^{-1}$. The energy spectrum of gamma-rays in the observed energy region from $0.8 \mathrm{TeV}$ to $7 \mathrm{TeV}$ is well described by the power law $I\left(>E_{\gamma}\right)=$ $(1.49 \pm 0.25) \times 10^{-12} \times E_{\gamma}^{-1.95 \pm 0.44}$. For each SNR the observation results are presented in Table 1 and Fig. 5. The spectral energy distribution of G166.0+4.3 by SHALON together with Fermi LAT [20] data is presented in Fig. 5 , top. Figure 5, bottom, presents $1420 \mathrm{MHz}$ radio contours of G166.0+4.3 from the CGPS [13] and X-ray 0.52 - $2.02 \mathrm{keV}$ image by ROSAT (RASS 3 processing) [19] compared to the TeV structure in energy range of $0.8-7$ TeV by SHALON. X-ray image is flattened and smoothed according to [19]. Four black contour levels are in logarithmic scale. The $\mathrm{TeV} \gamma$-ray emission regions correlate with the North-East and South-West parts of components visible in radio energies by CGPS (red contours in Fig. 5, bottom). TeV emission is more significant near the West, where the extended wing component and maximum of $\mathrm{X}$ ray emission viewed by ROSAT [19] are located (black contours in Fig. 5).

\section{Conclusion}

The observation results of Galactic shell-type supernova remnants at different evolution stages Cas A, Tycho's SNR, $\gamma$ Cygni SNR, IC 443 and G166.0+4.3 as well as remnant of classical nova GK Per by the SHALON mirror Cherenkov telescope are presented. The experimental data have confirmed the prediction of the discussed models and estimations about the hadronic generation mechanism of very high energy $\gamma$-rays in Tycho's SNR, Cas A, IC443 and $\gamma$ Cygni SNR. Further deeper investigations in high and very high energies are needed to better constrain astroparticle acceleration theory and model parameters.

\section{References}

[1] V.G. Sinitsyna and V.Yu. Sinitsyna, Astron. Lett. 37(9), 621 (2011)

[2] V.G. Sinitsyna and V.Yu. Sinitsyna "Astroparticle, Particle, Space Physics and Detectors Physics Applications" (Proc. 14th ICATPP, 2013) ed S Giani, C Leroy, P-G Rancoita, L Price and R Ruchti (Singapore: World Scientific 2014) vol 8 p 135

[3] A. A. Abdo et al., ApJ 710, L92 (2010)

[4] E.G. Berezhko et al. A\&A 400, 971 (2003)

[5] F.D. Seward et al., ApJ 652, 1277 (2006)

[6] V.G. Sinitsyna, et al. Nucl. Phys. B(Proc.Suppl.) 97, 215 (2001)

[7] T.-P. Li and Y.-Q. Ma, ApJ. 272, 317 (1983)

[8] J.S. Warren et al., ApJ. 634, 376 ( 2005).

[9] E.G. Berezhko, et al. A\&A 483, 529 (2008)

[10] D. Kosenko et al. A\&A 532, 114 (2011)

[11] T.A. Lozinskaya et al., Astron. Lett 26, 77 (2000)

[12] V.G. Sinitsyna, V.Yu. Sinitsyna, Bull. of the Lebedev Phys. Inst. 40(5), 113 (2013)

[13] A.R. Taylor et al., ApJ., 125, 3145 (2003).

[14] A. Weinstein, ArXiv:0912.4492.

[15] E. Aliu et al., ApJ 770, 93 (2013)

[16] I.V. Gosachinskij, Astron. Lett., 27, 233 (2001)

[17] A.A. Abdo, et al. ApJ. 712, 459 (2010)

[18] V.G. Sinitsyna and V.Yu. Sinitsyna, J. Phys.: Conf. Ser. 409, 012110 (2013)

[19] D.N. Burrows, Zh. Guo, ApJ. 421(No1), L19 (1994)

[20] M. Araya MNRAS 434, 2202 (2013) 